\title{
Análisis químico proximal (AQP) de productos de la colmena de un apiario de Tulancingo, Hidalgo.
}

\author{
Proximal Chemical Analysis of Hive Products from an Apiary of Tulancingo, Hidalgo \\ Gloria I. Camacho-Bernal ${ }^{a}$, Nelly del Socorro Cruz-Cansino $^{b}$, Ernesto Alanís-García $^{b}$, Zuli G. \\ Calderón-Ramos ${ }^{b}$, Esther Ramírez-Moreno ${ }^{b}$, Arianna Omaña-Covarrubias ${ }^{b}$.
}

\begin{abstract}
:
Since ancient times, the products of the beehive have been used as a medicinal remedy due to its nutritional and functional value established in various investigation through identification of compounds with important biological properties (Cornara, Biagi, Xiao, \& Burlando, 2017; Tenore, Ritieni, Campiglia, \& Novellino, 2012). The proximal chemical analysis (AQP), is an indispensable analytical method, carried out to determine the quality of a food product and includes the percentage of humidity, ash, fat (ethereal extract), protein and carbohydrates (Greenfield \& Southgate, 2003). The purpose of the present research was to evaluate the AQP of samples of honey, royal jelly, pollen and propolis. The samples were obtained from an apiary in Tulancingo, Hidalgo. The AQP was performed according to the procedures established by AOAC 2016, AOAC 2005, NMX-F-608-2011, NMX-F-089-S-1978. The results obtained showed that the products of the beehive analyzed are similar to previous studies and also comply with the guidelines established by Mexican and other countries regulations. Beehive products from the municipality of Tulancingo Hidalgo, are considered a good source of nutrients and energy, based on quality guidelines in international and national standards, in terms of composition and nutritional value.
\end{abstract}

Keywords:

Beehive products, proximal chemical analysis, AOAC

\section{Resumen:}

Desde la antigüedad, los productos de la colmena se han utilizado como remedio medicinal debido a su valor nutricional y funcional establecido en diversas investigaciones a través de la identificación de compuestos con propiedades biológicas importantes (Cornara, Biagi, Xiao, \& Burlando, 2017; Tenore, Ritieni, Campiglia, \& Novellino, 2012). El análisis químico proximal (AQP), es un método analítico indispensable, llevado a cabo para determinar la calidad de un producto alimenticio y comprende el porcentaje de humedad, ceniza, grasa (extracto etéreo), proteína e hidratos de carbono (Greenfield \& Southgate, 2003). El propósito de la presente investigación fue evaluar la proporción de humedad, cenizas, proteínas, grasa y carbohidratos de muestras de miel, jalea real, polen y propóleo. Las muestras se obtuvieron de un apiario de Tulancingo, Hidalgo. El AQP se realizó de acuerdo a los procedimientos establecidos por la AOAC 2016, AOAC 2005, NMX-F-608-2011, NMX-F-089-S-1978. Los resultados obtenidos muestran que los productos de la colmena analizados son similares a estudios previos y cumplen además con los lineamientos establecidos por las normativas mexicanas y de otros países. Los productos de la colmena originarios del municipio de Tulancingo Hidalgo, se consideran una buena fuente de nutrimentos y energía, en base a los lineamientos de calidad en normas internacionales y nacionales, en términos de composición y valor nutritivo.

\section{Palabras Clave:}




\section{Introducción}

Las abejas son insectos sociales considerados himenópteros (del orden Hymenoptera), pertenecen al género Apis. Actualmente se conocen dos especies domesticadas la Apis mellifera (de origen occidental, nativa de Europa, Asia, África e introducida en América) y la Apis cerana (del sur y sureste de Asia, dichas especies desde la antigüedad han despertado el interés sobre su consumo debido a su capacidad de producción y almacenamiento de miel y otras sustancias potencialmente útiles para los humanos (comúnmente denominados productos de la colmena) (Cornara, Biagi, Xiao, \& Burlando, 2017).

La miel, como el único edulcorante natural disponible en la antigüedad, fue un importante alimento para el homo sapiens desde sus comienzos, pues se sabe que la primera referencia escrita a la miel, remonta a 2000-2100 a. C, en ella se menciona su uso como un ungüento (Bogdanov, Jurendic, Sieber, \& Gallmann, 2013).Por definición, es la sustancia dulce producida por las abejas a partir del néctar de las flores o de las secreciones en plantas vivas, que las abejas recolectan, transforman y almacenan en panales (El Sohaimy, Masry, \& Shehata, 2015), es una solución compuesta al $80 \%$ por azúcares, principalmente fructosa (38\%) y glucosa (31\%) y en menor proporción sacarosa y maltosa (Cornara et al., 2017).

Su composición y sus atributos nutricionales varían de acuerdo a su origen geográfico y la temporada del año de donde provenga (Baroni et al., 2006; Marchini, Moreti, Otsuk, \& Sodré, 2007).

El polen por definición es la parte reproductora masculina de las plantas, el cual es recogido por las abejas trabajadoras en forma de gránulos y a su vez es el alimento recolectado para las abejas en crecimiento (Kaur, Kumar, \& Harjai, 2013).

La jalea real por su lado, es una secreción espesa y lechosa, producida por las mucosas glandulares hipo faríngeas y mandibulares de las abejas (Apis mellífera L.) que sirve como alimento de las larvas ((Isidorov, Czyzewska, Isidorova, \& Bakier, 2009). La abeja reina es alimentada con jalea real durante todo su período larvario, de ahí su nombre de "jalea real"(Srisuparbh, Klinbunga, Wongsiri, \& Sittipraneed, 2003).

Por último el propóleo, es la sustancia resinosa de las abejas forasteras (Apis meliifera $L$ ) derivado de la colecta de brotes y tejidos de plantas, después de mezclarlos con cera y polen resulta una sustancia maleable y compacta(Sun, Wu, Wang, \& Zhang, 2015). Es una resina preparada por las abejas para sellar las grietas, mantener la humedad y temperatura estable de la colmena (Huang, Zhang, Wang, Li, \& Hu, 2014).
El objetivo del presente trabajo fue evaluar la composición química (humedad, cenizas, proteínas, grasa y carbohidratos), de miel, jalea real, polen y propóleo de un apiario de Tulancingo, Hidalgo.

\section{Material y métodos}

Obtención de las muestras. Las muestras se obtuvieron de un apiario del municipio de Tulancingo, Hidalgo.

Determinación de humedad. Se realizó por el método de la "Association of Official Analytical Chemist (AOAC) (método 925.09; AOAC 2005), empleando un horno de secado (Scorpion Scientific, A52035, EUA) el cual consistió en tomar $1 \mathrm{gr}$ de muestra a peso constante a $105^{\circ} \mathrm{C}$ durante $4 \mathrm{~h}$, posteriormente se enfrió en desecador, durante $30 \mathrm{~min}$. El porcentaje de humedad se determinó de acuerdo a la (AOAC, 2005), por diferencia de peso empleando una balanza analítica (Adventurer modelo AR número 1140).

Determinación de cenizas. Se pesaron $5 \mathrm{~g}$ de muestra en cápsulas de porcelana a peso constante, para posteriormente pre-calcinar la muestra en una parrilla y llevarla a una calcinación total en la mufla (Thermolyne $\mathrm{F} 1500$, EUA) a $525^{\circ} \mathrm{C}$ durante $5-12 \mathrm{~h}$. Una vez frías las muestras se pesaron para determinar el porcentaje de cenizas conforme a la (AOAC, 2005, 2016) (AOAC, 2005, 2016).

Determinación de extracto etéreo. Para las muestras de polen y propóleo, la prueba se realizó conforme a la (AOAC, 2005; NMX-F-089-S-1978., 1978), utilizando el sistema de extracción de grasas (Buchi, B-811, Suiza). Se pesó $1 \mathrm{~g}$ de muestra en cartuchos de celulosa marca Whatman, posteriormente se añadió $80 \mathrm{~mL}$ de éter de petróleo a $30-75^{\circ} \mathrm{C}$ durante $3 \mathrm{~h}$, concluido dicho proceso se evaporó el éter sobrante, registrando el cambio de peso de los vasos y el resultado se expresó en porcentaje.

El resto de las muestras (miel clara, miel oscura y jalea), fueron analizadas por el método de Rosse-Gottlieb (UNAM, 2008).

Determinación de proteína cruda. Se realizó de acuerdo al método de Kjedahl para el análisis de nitrógeno total conforme la (AOAC, 2005; NMX-F-608-2011, 2011). Se empleó $1 \mathrm{~g}$ de muestra para la digestión con ácido sulfúrico; después de la digestión se llevó a un destilador automático. Destilada la muestra se valoró con ácido clorhídrico $0.1 \mathrm{~N}$. Se usó el factor 5.6 para polen y 6.25 para el resto de las muestras, el cual se multiplicó por el porcentaje de nitrógeno obtenido para el cálculo de proteína total.

Hidratos de carbono. Se determinó por diferencia de porcentaje (AOAC, 2005). 
Análisis estadístico. Todas las determinaciones se realizaron por triplicado. Se aplicó el Análisis de varianza (ANOVA) utilizando las medias \pm desviación estándar con prueba Tukey, para determinar diferencias significativas entre las muestras, utilizando un nivel de confianza del $95 \%(p<0.05)$, con el programa estadístico SPSS versión $22{ }^{\circledR}$.

\section{Resultados y discusión}

Humedad. El contenido de agua en los alimentos, la forma molecular y su localización dentro del producto alimenticio, son factores que afectan de modo significativo a características específicas como apariencia, textura, color, etc (Bello, 2000), se considera además un parámetro de calidad importante, sobre todo para la vida útil de los productos de la colmena (Adetuyi, Ibrahim, \& Ogundahunsi, 2009).

Los valores promedio con sus respectivas desviaciones estándar y las diferencias significativas $(p<0.05)$, entre cada producto de la colmena, se muestran en la Tabla 1 , se puede observar que el porcentaje mayor $(p<0.05)$ de humedad se encontró en la jalea real $(67.22 \pm 0.19 \%)$, este resultado concuerda con un estudio realizado en Italia, en 2009, el cual además menciona que la elevada humedad, se debe al constante suministro de la misma, en la colmena (panal) por parte de las abejas nodrizas y al esfuerzo en la colonia para mantener un nivel de humedad constante(Sanatini, Marcazzan, Caboni, Bogdanov, \& De Almaeida-Muradian, 2009).

Sin embargo es importante controlar la humedad en la miel por lo que existe una relación entre la proporción de humedad y el conteo de levaduras; ya que la miel tiene alto contenido de humedad, es más probable que fermente (Taylor et al., 1999). El porcentaje de humedad en las muestras de miel clara y miel oscura, cumplen con el establecido por la NMX-F036-1997 (NMX-F-036-1997, 1997), de un máximo de $20 \%$, de igual forma concuerdan con los obtenidos por Küçük (2007), (Küçük et al., 2007).

Cenizas. Las cenizas totales, representa el contenido de minerales en los alimentos. Su contenido en los productos analizados fluctuó entre $0.05 \%-1.7 \%$ (Tabla 1).

Los valores más altos significativamente $(p<0.05)$, se observaron en el polen $(1.7 \pm 0.11 \%)$, los cuales fueron similares a los obtenidos en un estudio realizado en Colombia(Fuenmayor B et al., 2014),

Por el contrario, los valores más bajos se encontraron en las muestras de miel clara $(0.29 \pm 0.17 \%)$ y miel oscura $(0.05 \pm 0.01 \%)$, sin embargo cumplen con lo establecido por la NMX-F-036-1997 y por normativas internacionales(Codex Alimentarius Commission, 2001), la cual establece un valor máximo de $0.6 \%$. Los resultados obtenidos para dicho parámetro son similares a los reportados por Omafubve en Nigeria en el 2009 (Omafuvbe \& Akanbi, 2009).
Extracto etéreo. En base a la Tabla 1 los valores para este parámetro oscilaron entre $4.43 \%-66.68 \%$ y una media de $18.52 \%$.

El porcentaje más alto significativamente $(p<0.05)$, se encontró en el propóleo (66.68 $1.89 \%)$, y se atribuye a su gran contenido de ceras el cual contiene terpenos, triterpenos, sequisterpenos volátiles, resinas y ácidos grasos(Huang et al., 2014).

Proteínas. La proporción de proteínas en las muestras se encontraron entre $0.36 \%$ y $17.26 \%$. Las diferencias significativas $(p<0.05)$ se muestran en la Tabla 1 . El aporte de proteínas en las mieles se encontró bajo (Tabla 1 ), sin embargo los valores fueron más altos en comparación a un estudio realizado por El Shohaimy et al., en el 2015, en el cual manifiesta que la variabilidad depende drásticamente del origen de la miel y al tipo de polen contenido en la miel.

El polen por el contrario, mostró un contenido de proteínas significativamente mayor $(p<0.05)$, en comparación al resto de las muestras (17.26 $\pm 0.47 \%)$, dichos valores pueden deberse a la gran variedad de aminoácidos que contiene(Nicolson, 2011). A pesar de que no hay una normativa mexicana que especifique los valores, caen dentro del rango establecido con la especificación regulatoria argentina que determina un valor de $15-28 \%$ del peso seco (Almeida-Muradian, Pamplona, Coimbra, \& Barth, 2005).

La proporción más baja se obtuvieron en las muestras de miel clara $(0.36 \pm 0.15 \%)$ y miel oscura $(0.37 \pm 0.11 \%)$, lo cual se puede explicar a su bajo contenido de polen, pues, de acuerdo con El Shoaimy y colaboradores, en 2015, el aporte de proteínas en la miel depende de la proporción de polen contenido en ella(El Sohaimy et al., 2015).

Hidratos de carbono. En la tabla 1, se muestran los valores para este parámetro en donde su contenido varió entre $13.64 \pm 0.91 \%$ (jalea real) y $78.38 \pm 0.26 \%$ (miel clara). La mayor proporción se encontró en las dos muestras de miel, esto se debe a que las mieles son azúcares compuestos principalmente por fructosa, glucosa, maltosa y sacarosa(Manyi-Loh, Clarke, \& Ndip, 2011; Oryan, Alemzadeh, \& Moshiri, 2016); seguido de ellas se encuentra el polen, en donde con respecto a las cifras obtenidas, se mostraron similares a un estudio realizado por Féas y colaboradores en 2012 (Feas, Vazquez-Tato, Estevinho, Seijas, \& Iglesias, 2012).

En el presente estudio los resultados en cuanto al contenido de carbohidratos en el polen de abeja $(60.21 \pm 1.05 \%)$, son similares a los obtenidos en un estudio realizado por Bertoncelj et al., en 2018, en donde se obtuvo un valor medio de $59.3 \%$ de hidratos de carbono (Bertoncelj, Pucihar, \& Borov, 2018). 
Tabla 1. Composición química proximal expresada en porcentaje de miel clara, miel oscura, jalea real, polen y propóleo

\begin{tabular}{|lccccc|} 
Muestra & Humedad & Cenizas & $\begin{array}{c}\text { Extracto } \\
\text { etéreo }\end{array}$ & Proteina & $\begin{array}{c}\text { Hidratos de } \\
\text { carbono }\end{array}$ \\
\hline Miel clara & $14.18 \pm 1.06^{\mathrm{c}}$ & $0.29 \pm 0.17^{\mathrm{d}}$ & $9.62 \pm 4.01^{\mathrm{b}}$ & $0.36 \pm 0.15^{\mathrm{d}}$ & $75.79 \pm 2.82^{\mathrm{a}}$ \\
\hline Miel oscura & $16.58 \pm 1.05^{\mathrm{b}}$ & $0.05 \pm 0.01^{\mathrm{d}}$ & $4.61 \pm 0.95^{\mathrm{c}}$ & $0.37 \pm 0.11^{\mathrm{d}}$ & $78.38 \pm 0.26^{\mathrm{a}}$ \\
\hline Jalea real & $67.22 \pm 0.19^{\mathrm{a}}$ & $0.710 \pm 0.08^{\mathrm{c}}$ & $4.43 \pm 0.35^{\mathrm{c}}$ & $13.99 \pm 0.41^{\mathrm{b}}$ & $13.64 \pm 0.91^{\mathrm{d}}$ \\
\hline Polen & $13.35 \pm 0.05^{\mathrm{c}}$ & $1.70 \pm 0.11^{\mathrm{a}}$ & $7.29 \pm 0.57^{\mathrm{b}, \mathrm{c}}$ & $17.26 \pm 0.47^{\mathrm{a}}$ & $60.21 \pm 1.05^{\mathrm{b}}$ \\
\hline Propóleo & $4.40 \pm 0.60^{\mathrm{d}}$ & $0.98 \pm 0.07^{\mathrm{b}}$ & $66.68 \pm 1.89^{\mathrm{a}}$ & $4.81 \pm 0.75^{\mathrm{c}}$ & $23.11 \pm 2.63^{\mathrm{c}}$ \\
\hline \multicolumn{5}{c}{${ }^{\mathrm{a}}$ Letras superíndices indican diferencias significativas entre las muestras. }
\end{tabular}

\section{Conclusiones}

A través de la realización del análisis químico proximal se pudieron resaltar la composición nutrimental de los productos de la colmena originarios del municipio de Tulancingo Hidalgo, como fuente de nutrimentos y energía, aportando datos que servirán de base para establecer el grado de cumplimiento de calidad de las muestras analizadas con respecto a normas internacionales y nacionales (como las establecidas en las Normas Oficiales Mexicanas), en términos de composición y valor nutritivo. De tal manera que al ser incluidos estos productos (miel, polen, jalea real y propóleo) como parte de la alimentación balanceada aportarían beneficios a la salud.

\section{Referencias}

1. Adetuyi, F. O., Ibrahim, T. A., \& Ogundahunsi, G. A. (2009). Total phenol, tocopherol and antibacterial quality of honey Apis mellifera sold in Owo community , Ondo State, Nigeria, 8(7), 1305-1309.

2. Almeida-Muradian, L. B., Pamplona, L. C., Coimbra, S., \& Barth, O. M. (2005). Chemical composition and botanical evaluation of dried bee pollen pellets. Journal of Food Composition and Analysis, 18(1), $105-111$.

3. AOAC. (2005). Official Methods of Analysis of AOAC International. (18th Ed). Gaithersburg, E.U.U.

4. AOAC. (2016). Official Methods of Analysis of AOAC International. (20 Ed.). Gaithersburg, E.U.U.

5. Baroni, M., Nores, M., Díaz, M., Chiabrando, G., Fassano, J., Costa, C., \& Wunderlin, D. (2006). Determination of volatile organic compound patterns characteristics of five unifloral honeys by solidphase microextraction-Gas chromatography-mass spectrometry coupled to chemimetrics. J. Agric. Food Chem., 54, 7235-7241.

6. Bello, J. (2000). Ciencia Bromatológica. Principios generales de los alimentos. España, Madrid: Ediciones Diaz de Santos.

7. Bertoncelj, J., Pucihar, T., \& Borov, A. K. (2018). Carbohydrate composition of Slovenian bee pollens. International Journal of Food and Technology, 53(8), 1-9. https://doi.org/10.1111/ijfs.13773

8. Bogdanov, S., Jurendic, T., Sieber, R., \& Gallmann, P. (2013). Honey for nutrition and health: A review. Journal of the American College of Nutrition, 27(6), 677-689.
9. Codex Alimentarius Commission. (2001). (2001a) Codex Standard for Honey, FAO, Rome. Alinorm 1: 19-26. Allinorm 1, 19-26.

10. Cornara, L., Biagi, M., Xiao, J., \& Burlando, B. (2017). Therapeutic properties of bioactive compounds from different honeybee products. Frontiers in Pharmacology, 8, 1-20.

11. El Sohaimy, S. A., Masry, S. H. D., \& Shehata, M. G. (2015). Physicochemical characteristics of honey from different origins. Annals of Agricultural Sciences, 60(2), 279-287.

12. Feas, X., Vazquez-Tato, M. P., Estevinho, L., Seijas, J. A., \& Iglesias, A. (2012). Organic bee pollen: Botanical origin, nutritional value, bioactive compounds, antioxidant activity and microbiological quality. Molecules, 17(7), 8359-8377.

13. Fuenmayor B, C., Zuluaga D, C., Díaz M, C., Quicazán de C, M., Cosio, M., \& Mannino, S. (2014). Evaluation of the physicochemical and functional properties of Colombian bee pollen. Rev.MVZ Córdoba, 19(1), 4003-4014.

14. Huang, S., Zhang, C.-P., Wang, K., Li, G., \& Hu, F.-L. (2014). Recent advances in the chemical composition of propolis. Molecules, 19, 19610-19632. https://doi.org/10.3390/molecules191219610

15. Isidorov, V. A., Czyzewska, U., Isidorova, A. G., \& Bakier, S. (2009). Gas chromatographic and mass spectrometric characterization of the organic acids extracted from some preparations containing lyophilized royal jelly. Journal of Chromatography B, 877(29), 3776-3780.

16. Kaur, R., Kumar, N. R., \& Harjai, K. (2013). Phytochemical analysis of different extracts of bee pollen. International Journal of Pharmaceutical and Biological Research, 4(3), 65-68.

17. Küçük, M., Kolayli, S., Karaoğlu, Ş., Ulusoy, E., Baltaci, C., \& Candan, F. (2007). Biological activities and chemical composition of three honeys of different types from Anatolia. Food Chemistry, 100(2), $526-534$

18. Manyi-Loh, C., Clarke, A., \& Ndip, R. (2011). An overview of honey: Therapeutic properties and contribution in nutrition and human health. African Journal of Microbiology Research, 5(9), 844-852.

19. Marchini, L., Moreti, A. C. de C. C., Otsuk, I., \& Sodré, G. (2007). Physicochemical composition of Apis mellifera honey samples from sao Paulo state, Brazil. Química Nova, 30(7), 1653-1657.

20. Nicolson, S. W. (2011). Bee Food: The chemistry and nutritional value of nectar, pollen and mixtures of the two. African Zoology, 46(2), 197-204.

21. NMX-F-036-1997. (1997). NMX F-036-1997.Alimentos-Miel ,especificaciones, métodos de prueba. Norma Oficial Mexicana.

22. NMX-F-089-S-1978. (1978). NMX-F-089-S-1978. DETERMINACIÓN DE EXTRACTO ETÉREO (MÉTODO SOXHLET) EN ALIMENTOS. Norma Oficial Mexicana, 1-3.

23. NMX-F-608-2011. (2011). NMX-F-608-2011. Alimentos. Determinación De Proteínas. Norma Oficial Mexicana, 3-6.

24. Omafuvbe, B. O., \& Akanbi, O. O. (2009). Microbiological and physico-chemical properties of some commercial Nigerian honey. African Journal Of Microbiology Research, 3(12), 891-896.25. Oryan, A., Alemzadeh, E., \& Moshiri, A. (2016). Biological properties and therapeutic activities of honey in wound healing: A narrative review and meta-analysis. Journal of Tissue Viability, 25(2), 98-118.

26. Sanatini, A. G., Marcazzan, G. L., Caboni, M. F., Bogdanov, S., \& De Almaeida-Muradian, L. B. (2009). Quality and standardisation of royal jelly. Journal of ApiProduct and ApiMedical Science, 1(1), 16.

27. Srisuparbh, D., Klinbunga, S., Wongsiri, S., \& Sittipraneed, S. (2003). Isolation and characterization of major royal jelly cDNAs and proteins of the honey bee (Apis cerana). Journal of Biochemistry and Molecular Biology, 36(6), 572-579. 
28. Sun, C., Wu, Z., Wang, Z., \& Zhang, H. (2015). Effect of ethanol/water solvents on phenolic profiles and antioxidant properties of Beijing propolis extracts. Evidence-Based Complementary and Alternative Medicine, 2015, 1-9.

29. Taylor, P., Bogdanov, S., Lüllmann, C., Martin, P., Ohe, W. Von Der Russmann, H., ... Vit, P. (1999). Bee World Honey quality and international regulatory standards: review by the International Honey Commission. Bee World, 80(261-69).

30. UNAM. (2008). Fundamentos y Técnicas de Análisis De Alimentos. México: Universidad Autónoma De México. 